\title{
COMPARISONS AND ASYMPTOTICS FOR EMPTY SPACE HAZARD FUNCTIONS OF GERM-GRAIN MODELS
}

\author{
GÜNTER LAST, ${ }^{*}$ Karlsruher Institut für Technologie \\ RYSZARD SZEKLI, ${ }^{* *}$ University of Wroctaw
}

\begin{abstract}
We study stochastic properties of the empty space for stationary germ-grain models in $\mathbb{R}^{d}$; in particular, we deal with the inner radius of the empty space with respect to a general structuring element which is allowed to be lower dimensional. We consider Poisson cluster and mixed Poisson germ-grain models, and show in several situations that more variability results in stochastically greater empty space in terms of the empty space hazard function. Furthermore, we study the asymptotic behaviour of the empty space hazard functions at 0 and at $\infty$.
\end{abstract}

Keywords: Germ-grain model; empty space hazard; relative distance; point process; Poisson cluster process; mixed Poisson process; hazard rate ordering

2010 Mathematics Subject Classification: Primary 60D05

Secondary 60G55

\section{Introduction}

The statistical analysis of a spatial pattern $Z \subset \mathbb{R}^{d}$ is based on the assumption that $Z$ is a random set in $\mathbb{R}^{d}$. Distance methods for point patterns usually begin by estimating nonparametric summary functions, such as the empty space function, nearest-neighbour distance distribution, Ripley's $K$-function, and derived statistics such as the pair correlation and the $J$-function. For a stationary spatial pattern $Z \subset \mathbb{R}^{d}$, which is not necessarily a point process, such as a germ-grain model, a particularly useful functional for estimating properties of $Z$ is the spherical contact distribution $H_{\mathrm{s}}\left(\right.$ of $Z$ ) and its hazard rate $h_{\mathrm{s}}$. To define these concepts, we consider the origin $0 \in \mathbb{R}^{d}$ under the condition that it is not covered by $Z$ and let a ball centred at 0 grow with unit speed until it hits $Z$ at some time $T>0$. Then $H_{\mathrm{s}}$ is the distribution of $T$ while $r_{\mathrm{s}}$ is its hazard rate. We follow [1] in calling $r_{\mathrm{s}}$ the empty space hazard of $Z$. Assume, for instance, that $Z$ is a Boolean model based on a stationary Poisson process $N$ of intensity $\lambda$ [18], [20]. Then $r_{\mathrm{s}}$ is a polynomial of degree $d-1$; see also (2.15) below. If $\tilde{Z}$ is a germ-grain model based on a stationary Poisson cluster process $\tilde{N}$ with intensity $\lambda$ and with the same typical grain as $Z$ (see Subsection 2.2 for more detail), then Proposition 3.3 implies that $\mathrm{P}(0 \in Z) \geq \mathrm{P}(0 \in \tilde{Z})$. Hence, the volume fractions of both models are ordered as expected. On the other hand, our Proposition 3.2 says in particular that $r_{\mathrm{s}}$ is greater than or equal to the empty space hazard $\tilde{r}_{\mathrm{s}}$ of $\tilde{Z}$. Hence, an observer, positioned at random in the complement of $Z$, sees less space around him than an observer in the complement of $\tilde{Z}$. It might come as a

Received 6 January 2011; revision received 12 May 2011.

This work was supported by MNiSW Research Grant N N201 394137.

* Postal address: Institut für Stochastik, Karlsruher Institut für Technologie, 76128 Karlsruhe, Germany.

Email address: guenter.last@kit.edu

** Postal address: Mathematical Institute, pl. Grunwaldzki 2/4, 50-384 Wrocław, Poland. 
surprise that this can be quantified in the strong sense of hazard rate ordering. Even if $\tilde{N} \neq N$, it is quite possible that $Z$ and $\tilde{Z}$ have the same volume fraction; see Remark 3.3. The above hazard rate ordering is also interesting in the case where the typical grain degenerates to a single point $x$. Then $Z=N+x, \tilde{Z}=\tilde{N}+x$, and $\mathrm{P}(0 \in Z)=\mathrm{P}(0 \in \tilde{Z})=0$.

Some early results on stochastic ordering of random closed sets can be found in [21], and a characterisation of the strong stochastic ordering of random closed sets is given in Theorem 1.4.42 of [15]. However, apart from Section 3.8 of [7] (dealing with volume fractions) and [12] (dealing with the spherical contact distribution of a Gauss-Poisson model), we are not aware of papers comparing functionals of stationary random closed sets. We shall compare here germ-grain models with the same germ intensity and the same typical grain in terms of empty space hazards. A common feature of our comparison results is that the models will be ordered with respect to a real-valued random or deterministic parameter. Increasing this parameter will not only increase the empty space hazard but also the variability of the underlying germ process. This means in a sense that a larger variance gives more empty space with more clustering. In fact, we will consider direction-dependent hazard rates and relative empty space hazards based on distances other than the Euclidean distance.

The main result on the asymptotic properties of empty space hazard rates is given in Theorem 3.2, which is based on Theorem 3.1. The main stochastic comparison results for empty space utilise two new stochastic relations which appear in a natural way in our study and which are easy to check for known distributions. We say that two random variables $\eta$ and $\tilde{\eta}$ taking values $0,1,2, \ldots$ are ordered in the length-biased probability generating functions ordering, and write $\eta<_{1-\mathrm{g}} \tilde{\eta}$ if the corresponding length-biased variables $\eta_{l}$ and $\tilde{\eta}_{l}$ are ordered in the probability generating ordering [19, Section 1.8], that is, $\mathrm{E}\left[s^{\eta_{l}}\right] \geq \mathrm{E}\left[s^{\tilde{\eta}_{l}}\right], s \in[0,1]$. For two nonnegative random variables $\Lambda$ and $\tilde{\Lambda}$, we say that they are ordered in the first cumulant order and write $\Lambda<$ cum $\tilde{\Lambda}$ if, for the corresponding cumulant generating functions $C_{\Lambda}$ and $C_{\tilde{\Lambda}}$ taking the first derivatives, we have $C_{\Lambda}^{\prime}(s) \geq C_{\tilde{\Lambda}}^{\prime}(s), s \leq 0$. We show for Neyman-Scott processes with cluster sizes $\eta$ and $\tilde{\eta}$ that $\eta<_{1-\mathrm{g}} \tilde{\eta}$ implies that the corresponding empty space distributions are ordered in the hazard rate ordering. For mixed Poisson germ-grain models, we show that if $\Lambda<$ cum $\tilde{\Lambda}$ then the corresponding empty space distributions are ordered in the hazard rate ordering.

The paper is organised as follows. Section 2 contains some preliminary material on empty space hazard germ-grain models and support measures, and concludes with a general theorem on the structure of relative empty space hazards. Section 3 contains our results on germgrain models based on Poisson cluster processes. After providing some general results on the structure and the asymptotic behaviour of (relative) empty space hazards, we shall compare Neyman-Scott and Gauss-Poisson germ-grain models. We also discuss the behaviour of cluster processes under scaling of the cluster points. Section 4 contains our results on mixed Poisson germ-grain models. We finish the paper with some concluding remarks.

\section{Preliminaries}

\subsection{Empty space hazard functions}

In this paper $Z$ will always denote a stationary random closed set in $\mathbb{R}^{d}$; see [15] and [18]. Let $\|x\|$ denote the Euclidean norm of a vector $x \in \mathbb{R}^{d}$, and let $d(x, A):=\inf \{\|x-y\|: y \in A\}$, the distance between $x \in \mathbb{R}^{d}$ and a set $A \subset \mathbb{R}^{d}$. Then the empty space distribution function $F$ of $Z$ is given by

$$
F(t)=\mathrm{P}(d(0, Z) \leq t), \quad t \geq 0,
$$


where 0 denotes the zero vector. The value $F(0)=\mathrm{P}(0 \in Z)$ is the volume fraction of $Z$. We can also write $F$ in terms of the capacity functional $T_{Z}$ (defined by $T_{Z}(K):=\mathrm{P}(Z \cap K \neq$ $\varnothing), K \subset \mathbb{R}^{d}$ compact), namely $F(t)=\mathrm{P}(Z \cap B(x, t) \neq \varnothing)=T_{Z}(B(x, t)), t \geq 0$, where $B(x, t)$ is the closed ball with centre $x$ and radius $t$. Stationarity of $Z$ ensures that this definition does not depend on $x$. Hence, $F(t)$ is the probability that $Z$ hits the ball $B(t):=B(0, t)$. There are many reasons for studying other distances than the Euclidean distance; see, e.g. [8]. For example, digital image analysers estimate the polygonal distance rather than the spherical distance. To quantify isotropy of point patterns, we need the elliptical distance. Since the distribution of $Z$ is not determined by $T_{Z}(B(x, t))$ for all balls, a larger class of sets provides better information on $Z$.

The usual way of introducing distances other than the spherical distance is to fix a structuring element (gauge body) $B \subset \mathbb{R}^{d}$. This is a compact convex set having $0 \in B$. Then the $B$-distance of a point $x \in \mathbb{R}^{d}$ to a set $K \subset \mathbb{R}^{d}$ is defined by

$$
d_{B}(x, K):=\inf \{t \geq 0:(x+t B) \cap K \neq \varnothing\} .
$$

It is possible that the set on the right-hand side is empty, e.g. if $B$ is lower dimensional. In such a case we set $d_{B}(x, K):=\infty$. Note that we have the translation invariance property $d_{B}(z+x, z+K)=d_{B}(x, K)$ for all $z \in \mathbb{R}^{d}$. Clearly, $d_{B}(x, K) \leq t$ if and only if $x$ is contained in the generalised outer parallel set $K+t B^{*}$ of $K$, where $B^{*}$ denotes the reflected set $\{-x: x \in B\}$. If $B$ is full dimensional (i.e. has a nonempty interior) and centrally symmetric (i.e. $B^{*}=B$ ), then $d_{B}(\cdot, \cdot)$ is a metric on $\mathbb{R}^{d}$ induced by the norm $d_{B}(\cdot, 0)$, and the pair $\left(\mathbb{R}^{d}, d_{B}\right)$ is called a Minkowski space.

If the $B$-distance $d_{B}(x, K)$ of a point $x \notin K$ is attained in a unique point $y$ in the boundary $\partial K$ of $K$ (i.e. if $\left.\left(x+d_{B}(x, K) B\right) \cap K=\{y\}\right)$, then we define the relative metric projection of $x$ on $K$ by $p_{B}(K, x):=y$, and the contact direction vector $u_{B}(K, x)$ as the element of $\partial B^{*}$ given by

$$
u_{B}(K, x):=\frac{x-y}{d_{B}(x, K)} .
$$

The points $x \in \mathbb{R}^{d} \backslash K$ for which the distance $d_{B}(x, K)$ is attained in more than one point of $K$ (and for which $u_{B}(x, K)$ is therefore not defined) form the exoskeleton $\operatorname{exo}_{B}(K)$ of $K$ (see [10]).

We define the (directed) $B$-relative empty space function $F_{B}$ of $Z$ by

$$
F_{B}(t, C):=\mathrm{P}\left(d_{B}(0, Z) \leq t, u_{B}(Z, 0) \in C\right), \quad t \geq 0, C \in \mathcal{B}^{d},
$$

where $\mathscr{B}^{d}$ is the system of Borel subsets of $\mathbb{R}^{d}$. Here we use the convention $u_{B}(Z, 0):=u_{0}$ if $0 \in Z$ or $d_{B}(0, Z)=\infty$, where $u_{0} \in \partial B^{*}$ is fixed. Definition (2.1) is subject to the assumption that the vector $u_{B}(Z, 0)$ is $\mathrm{P}$-almost surely (P-a.s.) well defined on $\{0<d(0, Z)<\infty\}$. If $B$ is strictly convex, containing 0 in its interior, then this is indeed the case. This follows from the fact that $\operatorname{exo}_{B}(Z)$ has volume 0 and from the stationarity of $Z$. (More general cases require a suitable assumption on the relative positions of $Z$ and $B$; see Subsection 2.2.) The function $F_{B}$ determines the joint distribution of the pair $\left(d_{B}(0, Z), u_{B}(Z, 0)\right)$, and, hence, that of the contact vector $d_{B}(0, Z) u_{B}(Z, 0)$. For each fixed $t$, the function $F_{B}(t, \cdot)$ is a measure on $\mathbb{R}^{d}$ concentrated on $\partial B^{*}$. The function $F_{B}(\cdot):=F_{B}\left(\cdot, \mathbb{R}^{d}\right)$ is called the (B-relative) empty space function of $Z$. The value $F_{B}(0)$ is the volume fraction of $Z$. In the case $F_{B}(0)<1$ the distribution function $H_{B}$ defined by $1-H_{B}(t):=\left(1-F_{B}(0)\right)^{-1}\left(1-F_{B}(t)\right)$ is the contact distribution function of $Z$ with respect to the structuring element $B$; see [14] and [20]. 
Using the Minkowski addition of sets $C, D \subset \mathbb{R}^{d}(C \oplus D:=\{x+y: x \in C, y \in D\})$ and stationarity, $F_{B}$ can be written as

$$
F_{B}(t)=V_{d}(A)^{-1} \mathrm{E}\left[V_{d}\left(\left(Z \oplus t B^{*}\right) \cap A\right)\right], \quad t \geq 0,
$$

for each Borel set $A$, such that the volume $V_{d}(A)$ of $A$ is positive and finite; see, e.g. [8] and [11]. Hansen et al. [8], utilising Federer's coarea theorem, showed that the empty space function $F_{B}$ of a random closed set $Z$ is absolutely continuous on $(0, \infty)$ with density

$$
f_{B}(t)=V_{d}(A)^{-1} \mathrm{E}\left[\int_{A \cap \partial\left(Z \oplus t B^{*}\right)}\left\|\nabla d_{B}(s, Z)\right\|^{-1} \mathcal{H}^{d-1}(\mathrm{~d} s)\right],
$$

where $\mathscr{H}^{i}, i \in\{0, \ldots, d\}$, denotes the $i$-dimensional Hausdorff measure on $\mathbb{R}^{d}, \partial A$ denotes the boundary of $A$, and $\nabla d_{B}$ denotes the gradient of the function $d_{B}$. In the Euclidean case, this formula reduces to

$$
f_{B(1)}(t)=V_{d}(A)^{-1} \mathrm{E}\left[\mathscr{H}^{d-1}(\partial(Z \oplus B(t)) \cap A)\right] .
$$

In this case the empty space hazard $r_{\mathrm{s}}$ (discussed in the introduction) equals the ratio of the expected measure of the boundary $\partial(Z \oplus B(t))$ inside the set $A$ to the volume of the space not occupied by $Z \oplus B(t)$ inside $A$. The direction-dependent (sub)distribution functions $F_{B}(\cdot, C)$, as defined in $(2.1)$, are also absolutely continuous on $(0, \infty)$ for any $C \in \mathscr{B}^{d}$. Letting $f_{B}(\cdot, C)$ denote its density, we define

$$
r_{B}(t, C):=\frac{f_{B}(t, C)}{1-F_{B}(t)},
$$

where $a / 0:=0$ for all $a \in \mathbb{R}$. We call the function $r_{B}:=r_{B}(\cdot, \cdot)$ the directed, $B$-relative empty space hazard of $Z$, and note that

$$
F_{B}(t)=1-\mathrm{P}(0 \notin Z) \exp \left[-\int_{0}^{t} r_{B}\left(u, \mathbb{R}^{d}\right) \mathrm{d} u\right] .
$$

We also note that $r_{B}(t, C)=h_{B}(t, C) /\left(1-H_{B}(t)\right)$, where $h_{B}(\cdot, C)$ is the density of the directional version of the contact distribution $H_{B}$.

Formulae (2.2) and (2.3) are the starting points for estimating these functions as well as their densities and hazard rates; see [1], [2], [4], [8], and [14]. Plots of these functions may serve as convenient summary statistics of a spatial pattern.

For fixed $B$, we shall order two random sets $Z$ and $\tilde{Z}$ with respect to their $B$-relative empty space hazard functions, and write

$$
Z<_{h-B} \tilde{Z}
$$

if and only if, for all $t \geq 0$ and $C \in \mathscr{B}^{d}$,

$$
r_{B}(t, C) \geq \tilde{r}_{B}(t, C)
$$

It can be seen from (2.4) that (2.6) (for $C=\mathbb{R}^{d}$ ) implies the usual (strong) stochastic ordering of contact distributions; see, e.g. [22, Section 1.4] for further details on such orderings. 


\subsection{Empty space hazard rates and support measures}

By a germ-grain model in $\mathbb{R}^{d}$ we mean a random set of the form

$$
Z=\bigcup_{n=1}^{\infty}\left(X_{n}+\xi_{n}\right)=\bigcup_{n=1}^{\infty}\left\{x+\xi_{n}: x \in X_{n}\right\},
$$

where the random points $\xi_{n}, n \in \mathbb{N}$, represent the locations of the germs and the primary grains $X_{n}, n \in \mathbb{N}$, are assumed to be random nonempty compact subsets of $\mathbb{R}^{d}$. We assume that the (simple) point process $N:=\left\{\xi_{n}: n \in \mathbb{N}\right\}$ is stationary, that is, the distribution of the shifted point process $N+x:=\left\{\xi_{n}+x: n \in \mathbb{N}\right\}$ does not depend on $x \in \mathbb{R}^{d}$, and that $N$ is independent of $\left(X_{n}\right)_{n \geq 1}$, which is a sequence of independent and identically distributed (i.i.d.) random sets. The intensity $\lambda_{N}:=\operatorname{E} \operatorname{card}\left\{n \in \mathbb{N}: \xi_{n} \in[0,1]^{d}\right\}$ of $N$ is assumed to be finite. An important special case is the Boolean model, where the germs are located according to a homogeneous Poisson process. Stationarity of $N$ entails that $Z$ is also stationary, i.e. that the distribution of $Z+x$ does not depend on $x$. It is convenient to denote by $X_{0}$ a typical grain having its distribution equal to that of $X_{n}$. We assume that $\mathrm{E}\left[V_{d}\left(X_{0}+K\right)\right]$ is finite for all compact $K \subset \mathbb{R}^{d}$. We will later use the fact that the capacity functional of a Boolean model is given by

$$
\mathrm{P}(Z \cap K \neq \varnothing)=1-\exp \left[-\lambda_{N} \mathrm{E}\left[V_{d}\left(X_{0}+K^{*}\right)\right]\right]
$$

In particular, the volume fraction of a Boolean model is given by

$$
\mathrm{P}(0 \in Z)=1-\exp \left[-\lambda_{N} \mathrm{E}\left[V_{d}\left(X_{0}\right)\right]\right] .
$$

A detailed introduction to germ-grain models can be found in [18] and [20].

Consider a convex, compact, and nonempty set $K \subset \mathbb{R}^{d}$. We assume that $K$ and $B^{*}$ are in general relative position, which means that $K$ and $B^{*}$ do not contain parallel segments in parallel and equally oriented support (hyper)planes. This means that $K$ and $B$ have independent support sets; see [18, p. 611] for more details. A sufficient condition is that $K$ or $B$ is strictly convex. This assumption guarantees that $p_{B}(K, x)$ (and, hence, $u_{B}(K, x)$ ) is defined for all $x \notin K$. Then there are finite measures $C_{0}(K ; B ; \cdot), \ldots, C_{d-1}(K ; B ; \cdot)$ on $\mathbb{R}^{d} \times \mathbb{R}^{d}$ which satisfy the local Steiner formula

$$
\begin{aligned}
V_{d}(\{x & \left.\left.\in \mathbb{R}^{d}: 0<d_{B}(x, K) \leq t,\left(p_{B}(K, x), u_{B}(K, x)\right) \in A \times C\right\}\right) \\
& =\sum_{i=0}^{d-1} t^{d-i} b_{d-i} C_{i}(K ; B ; A \times C)
\end{aligned}
$$

for all $A, C \in \mathcal{B}^{d}$, where $b_{i}(i \in \mathbb{N})$ denotes the volume of the unit ball in $\mathbb{R}^{i}$, and $b_{0}:=1$. These relative support measures of $K$ are uniquely determined by (2.9). They are concentrated on $\partial K \times \partial B^{*}$ and in fact on the relative normal bundle

$$
N_{B}(K):=\left\{\left(p_{B}(K, x), u_{B}(K, x)\right): x \notin K\right\}
$$

of $K$. If $B=B(1)$ then the measures $C_{i}(K ; \cdot):=C_{i}(K ; B(1) ; \cdot)$ are the generalised curvature measures of $K$. The total mass $V_{i}(K):=C_{i}\left(K ; \mathbb{R}^{d} \times \mathbb{R}^{d}\right)$ is the $i$ th intrinsic volume of $K$. In particular, $V_{d}(K)$ is the volume of $K, V_{d-1}(K)$ equals one half of the surface area, $V_{d-2}(K)$ 
is proportional to the integral mean curvature, $V_{1}(K)$ is proportional to the mean width of $K$, and $V_{0}(K)=1$. Equation (2.9) implies the classical Steiner formula

$$
V_{d}(K \oplus B(t))=\sum_{i=0}^{d} b_{d-i} t^{d-i} V_{i}(K) .
$$

In the general case, the total mass $C_{i}\left(K ; B ; \mathbb{R}^{d} \times \mathbb{R}^{d}\right)$ is a special mixed volume, namely,

$$
C_{i}\left(K ; B ; \mathbb{R}^{d} \times \mathbb{R}^{d}\right)=b_{d-i}^{-1}\left(\begin{array}{l}
d \\
i
\end{array}\right) V\left(K[i], B^{*}[d-i]\right), \quad i=0, \ldots, d-1 .
$$

For $i=0$, we have

$$
C_{0}\left(K ; B ; \mathbb{R}^{d} \times \mathbb{R}^{d}\right)=b_{d}^{-1} V_{d}(B) ;
$$

see [18] for the notation used here and for further details on support and curvature measures.

Consider now a germ-grain model $Z$ with convex, compact grains. In this paper it is always assumed that the reduced second moment measure of $N$ on $\mathbb{R}^{d}$ (see [5] and [20]), defined by

$$
\mathrm{E}\left[\sum_{\substack{x, y \in N \\ x \neq y}} \mathbf{1}\left\{x \in[0,1]^{d}, x-y \in \cdot\right\}\right]
$$

is absolutely continuous and that the typical grain $X_{0}$ and $B^{*}$ are a.s. in general relative position. It follows that $u_{B}(Z, 0)$ is a.s. well defined on $\left\{0<d_{B}(0, Z)<\infty\right\}$. This can be proved similarly to Proposition 4.9 of [9].

By the Steiner formula (2.10), our general integrability assumption on $X_{0}$ is equivalent to the finiteness of the mean intrinsic volumes $\mathrm{E} V_{i}\left(X_{0}\right), i \in\{0, \ldots, d\}$, of the typical grain. The Steiner formula (2.10) together with the local Steiner formula (2.9) imply that

$$
\bar{V}_{i, B}:=\mathrm{E} C_{i}\left(X_{0} ; B ; \mathbb{R}^{d} \times \mathbb{R}^{d}\right), \quad i=0, \ldots, d-1,
$$

are finite as well. Therefore, the mean relative support measures of the typical grain, defined by

$$
\bar{C}_{i, B}(\cdot):=\mathrm{E} C_{i}\left(X_{0} ; B ; \cdot\right), \quad i=0, \ldots, d-1,
$$

are finite measures on $\mathbb{R}^{d} \times \mathbb{R}^{d}$.

We further use the Palm probability $\mathrm{P}_{N}^{0}$ of $\mathrm{P}$ with respect to $N$ [5], [20]. We can interpret $\mathrm{P}_{N}^{0}(A)$ as the conditional probability of the event $A \in \mathcal{F}$ given that 0 is a 'randomly chosen point' of $N$. Let us define $X(x):=X_{n}$ if $x=\xi_{n}$ for some $n$, and $X(x):=\varnothing$ otherwise. Then, under $\mathrm{P}_{N}^{0},\{(x, X(x)): x \in N\}$ is an independently marked point process, i.e. conditionally on $N$, the grains $\{X(x): x \in N\}$ are independent and have the same distribution as $X_{0}$. For $\bar{V}_{i, B}>0, t \geq 0$, and $C \in \mathcal{B}^{d}$, let

$$
G_{i, B}(t, C):=\bar{V}_{i, B}^{-1} \int \mathbf{1}\{u \in C\} \mathrm{P}_{N}^{0}\left(d_{B}\left(y+t u, Z^{!}\right) \leq t\right) \bar{C}_{i, B}(\mathrm{~d}(y, u)),
$$

where

$$
Z^{!}:=\bigcup_{x \in N \backslash\{0\}}(X(x)+x)
$$


is the union of all grains except for the grain located at the origin. We set $G_{i, B} \equiv 0$ for $\bar{V}_{i, B}=0$. The function $G_{i, B}\left(\cdot, \mathbb{R}^{d}\right)$ can be interpreted as the distribution function of a random variable $\xi$, say, which can be constructed as follows. First select a point $Y$ of $N$ at random. Then sample a random element $(X, W)$ according to the distribution $\bar{V}_{i, B}^{-1} \bar{C}_{i, B}$. If $Y$ is not covered by $\bigcup_{x \in N \backslash\{Y\}}(X(x)+x)$ then $\xi$ is the $B$-distance from $Y+X$ to the exoskeleton $\operatorname{exo}_{B}(Z)$ in the direction $W$. Otherwise, $\xi=0$. We shall utilise the functions

$$
J_{i, B}(t, C):=\frac{G_{i, B}(\infty, C)-G_{i, B}(t, C)}{1-F_{B}(t)}, \quad i=0, \ldots, d-1,
$$

where $i \in\{0, \ldots, d-1\}, t \geq 0, C \subset \mathbb{R}^{d} \times \mathbb{R}^{d}$ is a Borel set, and

$$
G_{i, B}(\infty, C):=\lim _{t \rightarrow \infty} G_{i, B}(t, C)=\bar{V}_{i, B}^{-1} \bar{C}_{i, B}\left(\mathbb{R}^{d} \times C\right) .
$$

Special cases of these functions were introduced in [12, Section 5] after the point process case had been treated in [23]. The functions $J_{i, B}(t, C)$ can be used as nonparametric measures for expressing differences between a general germ-grain model and Boolean model with the same values of $\lambda_{N} \bar{V}_{i, B}$. Intuitively speaking, such measures detect interactions and clustering effects. In the Euclidean case (and for $C=\mathbb{R}^{d}$ ) the following theorem was proved in [12] and [13]. The present relative version is implicit in [9], at least in the case of a strictly convex $B$. The general result can be derived from Theorem 5.1 of [10].

Theorem 2.1. Consider a stationary germ-grain model satisfying the assumptions formulated above. Then the B-relative empty space hazard is given by

$$
r_{B}(t, C)=\sum_{i=0}^{d-1}(d-i) t^{d-i-1} b_{d-i} \lambda_{N} \bar{V}_{i, B} J_{i, B}(t, C) .
$$

If $N$ is a Poisson process (i.e. $Z$ is a Boolean model with convex grains) then Slivnyak's theorem (see, e.g. [18, Theorem 3.5.9] and [20, p. 121]) implies that

$$
\mathrm{P}_{N}^{0}\left(d_{B}\left(y+t u, Z^{!}\right)>t\right)=1-F_{B}(t)
$$

Hence, (2.14) simplifies to

$$
r_{B}(t, C)=\sum_{i=0}^{d-1}(d-i) b_{d-i} t^{d-i-1} \lambda_{N} \bar{S}_{i, B}(C),
$$

where $\bar{S}_{i, B}(C):=\bar{C}_{i, B}\left(\mathbb{R}^{d} \times C\right)$. In the case of a strictly convex gauge body $B$ this result can be found in [9]. Note that in the Boolean model $r_{B}\left(\cdot, \mathbb{R}^{d}\right)$ is determined by the intensity $\lambda_{N}$ and the mean mixed volumes $\bar{V}_{1, B}, \ldots, \bar{V}_{d-1, B}$ of $X_{0}$. For $d=2$ and $B=B(1)$, for instance, the only parameter of $X_{0}$ influencing the empty space hazard is its mean boundary length $\bar{V}_{1}$.

Although the functions $J_{i, B}$ occurring in (2.14) cannot be explicitly calculated in more general cases, the formula provides deeper insight into the structure of relative empty space hazards. Moreover, Theorem 2.1 and its consequences for more specific models (see Theorem 3.1 and Proposition 4.1) is our main tool for proving the asymptotic and comparison results in the next sections. 


\section{Poisson cluster models}

In this section we shall consider germ-grain models with the Poisson cluster point process as a germ process. Recall from [5, Chapter 6.3] that a (stationary) Poisson cluster point process $N$ can be written as

$$
N=\bigcup_{x \in \Pi}\left(L_{x}+x\right)
$$

where $\Pi$ is a Poisson process with positive and finite intensity $\lambda_{\Pi}$, and the family $\left\{L_{x}: x \in \Pi\right\}$ consists of finite random point processes on $\mathbb{R}^{d}$. Given $\Pi$, the family $\left\{L_{x}: x \in \Pi\right\}$ is i.i.d. with the same distribution as a typical cluster $L_{0}$. We assume that

$$
\gamma:=\mathrm{E} \operatorname{card} L_{0}
$$

is finite and positive; hence, $\lambda_{N}=\lambda_{\Pi} \gamma$. By stationarity we have $\mathrm{E}[N(B)]=\lambda_{N} V_{d}(B)$ for all $B \in \mathcal{B}^{d}$. It is a well-known fact (see [5, Equation (6.3.11)]) that

$$
\mathrm{E}\left[N(B)^{2}\right]=(\mathrm{E}[N(B)])^{2}+\lambda_{\Pi} \mathrm{E}\left[\int L_{0}(B-x)^{2} \mathrm{~d} x\right], \quad B \in \mathscr{B}^{d} .
$$

Example 3.1. Assume that

$$
L_{0}= \begin{cases}\varnothing & \text { if } \eta=0, \\ \left\{Y_{i}: i=1, \ldots, n\right\} & \text { if } \eta=n \geq 1,\end{cases}
$$

where the random cluster size $\eta \geq 0$, and the random vectors $Y_{i}, i=1,2, \ldots$, are independent. Assume also that the $Y_{i}$ have the same (cluster point) distribution $V$, say. Then $N$ is called the Neyman-Scott process. We always assume that $\gamma=\mathrm{E}[\eta]$ is positive and finite.

Example 3.2. Let $\eta$ be a $\{1,2\}$-valued random variable, and assume that

$$
L_{0}= \begin{cases}\{0\} & \text { if } \eta=1, \\ \{0, Y\} & \text { if } \eta=2,\end{cases}
$$

where $Y$ is a random vector independent of $\eta$. Then $N$ is called the Gauss-Poisson process. In this case a cluster $L_{0}+x$ associated with a parent point $x$ say, contains $x$ and, with probability $p:=\mathrm{P}(\eta=2)$, also a secondary point $Y+x$. Note that the mean cluster size is given by $\gamma=1+p$.

We assume that the reduced second moment measure

$$
\mathrm{E}\left[\sum_{\substack{x, y \in L_{0} \\ x \neq y}} 1\{x-y \in \cdot\}\right]
$$

of $L_{0}$ is absolutely continuous on $\mathbb{R}^{d}$. The well-known second-order properties of Poisson cluster point processes (see, e.g. [5]) easily imply that the measure defined at (2.11) is absolutely continuous as well. For the Neyman-Scott process of Example 3.1, our assumption on $L_{0}$ is implied by the absolute continuity of the cluster point distribution $V$. For a Gauss-Poisson process, it is sufficient to assume that the (secondary) point $Y$ has an absolutely continuous distribution. 
We consider the germ-grain model $Z$ based on the Poisson cluster process $N$ and typical grain $X_{0}$. We fix a gauge body $B$ and recall our general assumptions made after (2.11). Alternatively, we shall treat $Z$ as a Boolean model with nonconvex grains which are

$$
\bigcup_{y \in L_{x}}(X(y)+y), \quad x \in \Pi .
$$

In the degenerate case $X_{0}=\{0\}$ the random closed set $Z$ coincides with $N$. In this case it is no loss to assume that $B$ has a nonempty interior, because otherwise $F_{B}(t)=r_{B}(t)=0$ for all $t \geq 0$.

For a given finite point pattern $\psi=\left\{x_{n}: n=1, \ldots, m\right\}$, denote by $\Gamma(\psi, \cdot)$ the distribution of the random closed set $\bigcup_{n=1}^{m}\left(X_{n}+x_{n}\right)$, where $X_{1}, \ldots, X_{m}$ are independent with distribution equal to that of $X_{0}$. We also set $\Gamma(\varnothing, \cdot):=\delta_{\varnothing}$. Let $Y_{0}$ be a random closed set with distribution

$$
\mu(\cdot):=\frac{1}{\gamma} \mathrm{E}\left[\sum_{y \in L_{0}} \int \mathbf{1}\{A \in \cdot\} \Gamma\left(\left(L_{0}-y\right) \backslash\{0\}, \mathrm{d} A\right)\right] .
$$

This probability measure describes the distribution of the finite germ-grain model associated with a typical cluster as seen from a randomly chosen cluster point, after removing the grain around the chosen point. We assume that $Y_{0}$ and the typical grain $X_{0}$ are independent, and define, for $i \in\{0, \ldots, d-1\}, t \geq 0$, and a Borel set $C \subset \mathbb{R}^{d}$,

$$
K_{i, B}(t, C):=\frac{1}{\bar{V}_{i, B}} \mathrm{E}\left[\int \mathbf{1}\left\{d_{B}\left(x+t u, Y_{0}\right)>t, u \in C\right\} C_{i}\left(X_{0} ; B ; \mathrm{d}(x, u)\right)\right],
$$

provided that $\bar{V}_{i, B}>0$. Otherwise, we set $K_{i, B} \equiv 0$. The following proposition yields an explicit formula for the $B$-relative empty space hazard of a Poisson cluster germ-grain model, this time in terms of $X_{0}$ and $Y_{0}$ locally describing a cluster.

Theorem 3.1. The B-relative empty space hazard of a Poisson cluster germ-grain model with compact, convex grains is given by

$$
r_{B}(t, C)=\sum_{i=0}^{d-1}(d-i) t^{d-i-1} b_{d-i} \lambda_{N} \bar{V}_{i, B} K_{i, B}(t, C) .
$$

Proof. Our aim is to use (2.14). To do so, we recall that the Palm probability measure $\mathrm{P}_{N}^{0}$ of a Poisson cluster process $N$ satisfies

$$
\mathrm{P}_{N}^{0}(N \in \cdot)=\mathrm{E}\left[\int \mathbf{1}\{N \cup \psi \in \cdot\} Q_{L_{0}}^{0}(\mathrm{~d} \psi)\right]
$$

where

$$
Q_{L_{0}}^{0}(\cdot):=\gamma^{-1} \mathrm{E}\left[\sum_{y \in L_{0}} 1\left\{L_{0}-y \in \cdot\right\}\right]
$$

is the Palm distribution of the typical cluster $L_{0}$; see, e.g. [20]. As in Last and Holtmann [12], this implies that

$$
\mathrm{P}_{N}^{0}\left(d_{B}\left(x+t u, Z^{!}\right)>t\right)=\left(1-F_{B}(t)\right) \iint \mathbf{1}\left\{d_{B}(x+t u, A)>t\right\} \Gamma(\psi \backslash\{0\}, \mathrm{d} A) Q_{L_{0}}^{0}(\mathrm{~d} \psi) .
$$


By definition (2.12) and the definition of $Y_{0}$, this means that

$$
\frac{G_{i, B}(\infty, C)-G_{i, B}(t, C)}{1-F_{B}(t)}=\frac{1}{\bar{V}_{i, B}} \mathrm{E}\left[\int \mathbf{1}\left\{d_{B}\left(x+t u, Y_{0}\right)>t\right\} \mathbf{1}\{u \in C\} \bar{C}_{i, B}(\mathrm{~d}(x, u))\right] .
$$

By definition (3.4), the above right-hand side equals $K_{i, B}(t, C)$. Inserting this into (2.14) gives the asserted equation (3.5).

Let $v_{B}$ be the measure on $\mathbb{R}^{d}$ given by

$$
v_{B}(\cdot):=d \int_{B^{*}} \mathbf{1}\left\{\frac{x}{d_{B}(0, x)} \in \cdot\right\} \mathrm{d} x,
$$

where $d_{B}(0, x):=d_{B}(0,\{x\})$. This is the null measure if and only if $B$ has an empty interior.

Corollary 3.1. The B-relative empty space hazard of the Poisson cluster point process $N$ is given by

$$
r_{B}(t, C)=\lambda_{\Pi} t^{d-1} \int_{C} \mathrm{E}\left[\int_{\mathbb{R}^{d}} 1\left\{\left(\left(L_{0}-x\right) \backslash\{0\}\right) \cap(t u+t B)=\varnothing\right\} L_{0}(\mathrm{~d} x)\right] v_{B}(\mathrm{~d} u) .
$$

In particular,

$$
r_{B(1)}(t, C)=\lambda_{\Pi} t^{d-1} \int_{C} \mathrm{E}\left[\int_{\mathbb{R}^{d}} \mathbf{1}\left\{\left(\left(L_{0}-x\right) \backslash\{0\}\right) \cap B(t u, t)=\varnothing\right\} L_{0}(\mathrm{~d} x)\right] \mathscr{H}^{d-1}(\mathrm{~d} u) .
$$

Proof. Computing the left-hand side of (2.9) for $K=\{0\}$ and $A=\{0\} \times C$ easily shows that

$$
d b_{d} C_{0}(\{0\} ; B ;\{0\} \times \cdot)=v_{B}(\cdot)
$$

and that $C_{i}(\{0\} ; B ; \cdot)=0$ for $i \geq 1$. Result (3.6) is then a consequence of Theorem 3.1. If $B=B(1)$ then $v_{B}$ is the $(d-1)$-dimensional Hausdorff measure on the unit sphere $S^{d-1}:=$ $\partial B(1)$. Therefore, (3.6) implies (3.7).

If $L_{0}=\{0\}$, we have $N=\Pi$ and (3.6) simplifies to

$$
r_{B}(t, C)=\lambda_{N} t^{d-1} v_{B}(C),
$$

in accordance with (2.15).

Example 3.3. Assume that $N=Z$ is a Neyman-Scott process as defined in Example 3.1. From (3.6) and a straightforward calculation,

$$
r_{B}(t, C)=\lambda_{\Pi} t^{d-1} \int_{\mathbb{R}^{d}} \int_{\mathbb{R}^{d}} g^{\prime}\left(\mathrm{P}\left(Y_{1}-x \notin t u+t B\right)\right) V(\mathrm{~d} x) v_{B}(\mathrm{~d} u),
$$

where $g^{\prime}$ is the derivative of the probability generating function $g$ of $\eta$. This result generalises Equation (30) of [11].

Example 3.4. Assume that $N=Z$ is a Gauss-Poisson process as defined in Example 3.2. Then

$$
\begin{aligned}
\left(\lambda_{\Pi}\right)^{-1} t^{1-d} r_{B}(t, C)= & (1-p) v_{B}(C)+p \int_{C} \mathrm{P}(Y \notin t u+t B) v_{B}(\mathrm{~d} u) \\
& +p \int_{C} \mathrm{P}(-Y \notin t u+t B) v_{B}(\mathrm{~d} u)
\end{aligned}
$$


Our next proposition deals with the asymptotic behaviour of $r_{B}(t, C)$ as $t \rightarrow 0$ or $t \rightarrow \infty$, respectively. The tangential cone (or support cone) $T(B, u)$ of $B$ at $u \in B$ is the closure of $T^{\prime}(B, u):=\{t(x-u): t>0, x \in B\} ;$ see [17].

Theorem 3.2. Assume that there is some $j \in\{0, \ldots, d-1\}$ such that $\bar{V}_{i}=0$ for $i>j$. Let $C \in \mathscr{B}^{d}$. Then $t^{j+1-d} r_{B}(t, C)$ is monotone increasing in $t$. Moreover,

$$
\begin{gathered}
\lim _{t \rightarrow 0} t^{j+1-d} r_{B}(t, C)=(d-j) b_{d-j} \lambda_{N} \mathrm{E}\left[\int_{\mathbb{R}^{d} \times C} \mathbf{1}\left\{x \notin Y_{0}\right\} C_{d-j}\left(X_{0} ; B ; \mathrm{d}(x, u)\right)\right], \\
\lim _{t \rightarrow \infty} t^{1-d} r_{B}(t, C)=d b_{d} \lambda_{N} \mathrm{E}\left[\int_{\mathbb{R}^{d} \times C} \mathbf{1}\left\{(x+T(B,-u)) \cap Y_{0}=\varnothing\right\}\right. \\
\left.\times C_{0}\left(X_{0} ; B ; \mathrm{d}(x, u)\right)\right] .
\end{gathered}
$$

Proof. The first assertion follows from (3.5) upon observing that $d_{B}\left(x+t u, Y_{0}\right)>t$ is equivalent to $(x+t u+t B) \cap Y_{0}=\varnothing$. Relation (3.9) follows from monotone convergence. Monotone convergence also shows that

$$
\lim _{t \rightarrow \infty} t^{1-d} r_{B}(t, C)=d b_{d} \lambda_{N} \mathrm{E}\left[\int_{\mathbb{R}^{d} \times C} \mathbf{1}\left\{\left(x+T^{\prime}(B,-u)\right) \cap Y_{0}=\varnothing\right\} C_{0}\left(X_{0} ; B ; \mathrm{d}(x, u)\right)\right] .
$$

Since measure (3.2) is absolutely continuous, it follows that

$$
\mathrm{P}\left(x+T^{\prime}(B,-u) \cap Y_{0}=\varnothing, x+T(B,-u) \cap Y_{0} \neq \varnothing\right)=0 .
$$

This implies (3.10).

Remark 3.1. One might wonder whether the right-hand side of (3.10) is positive for $C=\mathbb{R}^{d}$. A simple sufficient condition is that $\mathrm{P}\left(\operatorname{card} L_{0}=1\right)>0$, because in this case $Y_{0}$ is, with positive probability, empty. Another sufficient condition is to assume that $B$ is smooth (any boundary point has a unique supporting hyperplane) and that the diameter of the typical grain can take arbitrarily small positive values with positive probability. These assumptions would allow us to apply the method of [11, Section 6.5] on a set of positive probability. We do not go into further details.

Remark 3.2. The second assertion of Theorem 3.2 shows in particular that $F_{B}$ is light tailed, i.e. has a finite exponential moment.

Next we deal with the special case $Z=N$. For small values of $t$, the empty space hazard of a Poisson cluster point process behaves approximately like the empty space hazard of a Poisson process with the same intensity; cf. (3.8). For large values of $t$, the asymptotic behaviour is the same as that of the Poisson process $\Pi$ thinned at points $x$ where $L_{x}$ is empty, for which the intensity equals $\mathrm{P}\left(L_{0} \neq \varnothing\right) \lambda_{\Pi}$. This means in a sense that points in clusters cannot be distinguished from a very far distance, irrespective of any specific assumptions on the typical cluster $L_{0}$. This generalises Equation (22) of [11]. A weaker version of this latter result has been rediscovered by Bordenave and Torrisi [3].

Corollary 3.2. The B-relative empty space hazard of a Poisson cluster point process satisfies

$$
\begin{aligned}
& \lim _{t \rightarrow 0} t^{1-d} r_{B}(t, C)=\lambda_{N} d v_{B}(C), \\
& \lim _{t \rightarrow \infty} t^{1-d} r_{B}(t, C)=\mathrm{P}\left(L_{0} \neq \varnothing\right) \lambda_{\Pi} v_{B}(C) .
\end{aligned}
$$


Proof. By Theorem 3.2, it remains to prove (3.11). Convergence (3.10) and definition (3.3) of the distribution of $Y_{0}$ imply that

$$
\lim _{t \rightarrow \infty} t^{1-d} r_{B}(t, C)=\lambda_{\Pi} \int_{C} \mathrm{E}\left[\int \mathbf{1}\left\{\left(\left(L_{0}-x\right) \backslash\{0\}\right) \cap T(B,-u)=\varnothing\right\} L_{0}(\mathrm{~d} x)\right] v_{B}(\mathrm{~d} u) .
$$

Let us fix for a moment a regular boundary point $u$ of $B^{*}$. This means that $B$ has a unique supporting hyperplane at $-u$; see [17]. Then $T(B,-u)$ is the supporting half-space of $B$ at $-u$; see Section 2.2 of [17]. It now follows as in [11, Section 6.5] that

$$
\mathrm{E}\left[\int \mathbf{1}\left\{\left(\left(L_{0}-x\right) \backslash\{0\}\right) \cap T(B,-u)=\varnothing\right\} L_{0}(\mathrm{~d} x)\right]=\mathrm{P}\left(L_{0} \neq \varnothing\right) .
$$

It remains to note that $v_{B}$-almost all $u \in \mathbb{R}^{d}$ are regular boundary points of $B^{*}$. This follows from the fact that $v_{B}$ is absolutely continuous with respect to a $(d-1)$-dimensional Hausdorff measure on $\partial B^{*}$ and Theorem 2.2.4 of [17].

Now we shall compare the $B$-relative empty space hazard $r_{B}$ of $Z$ with that of another Poisson cluster germ-grain model $\tilde{Z}$ with the same intensity $\lambda$ of germs and the same typical grain $X_{0}$. Both underlying germ processes are assumed to satisfy the technical assumption formulated at (3.2). We denote the characteristics of $\tilde{Z}$ by $\tilde{\Pi}, \tilde{L}_{0}, \tilde{K}_{i, B}, \tilde{r}_{B}$, etc. We begin with a direct consequence of Proposition 3.1.

Proposition 3.1. Let $C \in \mathcal{B}^{d}$ and $t \geq 0$ be such that

$$
K_{i, B}(t, C) \geq \tilde{K}_{i, B}(t, C), \quad i=0, \ldots, d-1 .
$$

Then (2.6) holds.

Proposition 3.1 implies that the relative empty space hazard of a Boolean model is always greater than that of a Poisson cluster germ-grain model having the same germ intensity and the same typical grain.

Proposition 3.2. Assume that $Z$ is a Boolean model with typical convex, compact grains distributed as $X_{0}$, and that $\tilde{Z}$ is a Poisson cluster germ-grain model with equal intensity, and also with typical grains distributed as $X_{0}$. Then $Z<_{h-B} \tilde{Z}$.

Proof. Since $Z$ is a Boolean model, we have $L_{0}=\{0\}$ and $\gamma=1$. Hence, if $\bar{V}_{i, B}>0$,

$$
K_{i, B}(t, C)=\bar{V}_{i, B}^{-1} \bar{C}_{i, B}\left(\mathbb{R}^{d} \times C\right) \geq \tilde{K}_{i, B}(t, C) .
$$

While a Boolean model has stochastically smaller empty space than a related Poisson cluster germ-grain model, it has a greater volume fraction. Under a different set of assumptions (more specific Poisson cluster processes and deterministic but possibly nonconvex grains), the result was proved in Section 3.8 of [7].

Proposition 3.3. Under the assumptions of Corollary 3.2,

$$
\mathrm{P}(0 \in Z) \geq \mathrm{P}(0 \in \tilde{Z}) .
$$

Proof. The volume fraction of $Z$ is given by (2.8). On the other hand, $\tilde{Z}$ is also a Boolean model, but based on the Poisson process $\tilde{\Pi}$ and with a typical (possibly nonconvex) grain

$$
\tilde{X}_{0}:=\bigcup_{x \in \tilde{L}_{0}} X(x)+x
$$


where $\tilde{L}_{0}$ is the typical cluster associated with $\tilde{Z}$ and, given $\tilde{L}_{0}$, the family $\left\{X(x): x \in \tilde{L}_{0}\right\}$ consists of independent random closed sets with the same distribution as $X_{0}$. Therefore, we obtain, from (2.8),

$$
\mathrm{P}(0 \in \tilde{Z})=1-\exp \left[-\lambda_{\tilde{\Pi}} \mathrm{E} V_{d}\left(\tilde{X}_{0}\right)\right]
$$

We have

$$
\mathrm{E} V_{d}\left(\tilde{X}_{0}\right) \leq \mathrm{E} \sum_{x \in \tilde{L}_{0}} V_{d}(X(x))=\mathrm{E} \sum_{x \in \tilde{L}_{0}} \mathrm{E}\left[V_{d}(X(x)) \mid \tilde{L}_{0}\right]=\gamma \bar{V}_{d} .
$$

Inserting this into (3.13) and comparing with (2.8) yields the assertion.

Remark 3.3. Consider the hypothesis of Proposition 3.3. The proof of this proposition shows that inequality (3.12) becomes an equality if and only if

$$
V_{d}(X(x) \cap X(y))=0, \quad x, y \in \tilde{L}_{0}, x \neq y, \mathrm{P} \text {-a.s. }
$$

This is, for instance, the case if the cluster points have minimal distance $2 t_{0}$ from each other for some $t_{0}>0$, and $X_{0}$ is a.s. contained in the ball $B\left(t_{0}\right)$.

We continue with ordering results for Neyman-Scott processes. Let $\eta$ and $\tilde{\eta}$ be two counting variables (i.e. taking values in $\{0,1,2, \ldots\}$ ), and let $\eta_{l}$ be the (shifted) length-biased version of $\eta$. This means that $\eta_{l}$ has distribution $\mathrm{E}[\eta]^{-1} \mathrm{E}[\eta \mathbf{1}\{\eta-1 \in \cdot\}]$. Denoting by $\tilde{\eta}_{l}$ the lengthbiased version of $\tilde{\eta}$, we define the length-biased probability generating functions ordering

$$
\eta<1-\mathrm{g} \tilde{\eta}
$$

by

$$
\mathrm{E}\left[s^{\eta_{l}}\right] \geq \mathrm{E}\left[s^{\tilde{\eta}_{l}}\right], \quad s \in[0,1] .
$$

This means that $\eta_{l}$ is smaller than $\tilde{\eta}_{l}$ in the generating function order (see [19, Section 1.8]). Note that $\eta<_{1-\mathrm{g}} \tilde{\eta}$ is equivalent to

$$
\mathrm{E}[\eta]^{-1} \mathrm{E}\left[\mathbf{1}\{\eta \geq 1\} \eta a^{\eta-1}\right] \geq \mathrm{E}[\tilde{\eta}]^{-1} \mathrm{E}\left[\mathbf{1}\{\tilde{\eta} \geq 1\} \tilde{\eta} a^{\tilde{\eta}-1}\right], \quad a \in[0,1] .
$$

Another way of expressing this relation is by the generating functions of $\eta$ and $\tilde{\eta}$. Denote by

$$
g_{\eta}(s):=\mathrm{E}\left[s^{\eta}\right], \quad s \in[0,1],
$$

the probability generating function of $\eta$. Then (3.14) means that

$$
\mathrm{E}[\eta]^{-1} g_{\eta}^{\prime}(s) \geq \mathrm{E}[\tilde{\eta}]^{-1} g_{\tilde{\eta}}^{\prime}(s), \quad s \in[0,1] .
$$

Relation (3.14) does not imply that the corresponding means $\mathrm{E}[\eta]$ and $\mathrm{E}[\tilde{\eta}]$ are ordered. For example, if $\eta \equiv 1, \eta_{1} \equiv 2$, and $\eta_{2}$ equals 0 with probability $\frac{1}{2}$ and 1 with probability $\frac{1}{2}$, then $\eta<_{1-\mathrm{g}} \eta_{1}$ and $\eta<_{1-\mathrm{g}} \eta_{2}$, but $\mathrm{E}[\eta]=1<\mathrm{E}\left[\eta_{1}\right]=2$ and $\mathrm{E}[\eta]=1>\mathrm{E}\left[\eta_{2}\right]=\frac{1}{2}$. If $\eta$ and $\tilde{\eta}$ have a finite second moment, then (3.15) implies that

$$
\mathrm{E}[\eta]^{-1} \mathrm{E}\left[\eta^{2}\right] \leq \mathrm{E}[\tilde{\eta}]^{-1} \mathrm{E}\left[\tilde{\eta}^{2}\right] .
$$

If, moreover, $\mathrm{E}[\eta]=\mathrm{E}[\tilde{\eta}]$ then this implies that $\operatorname{var}[\eta] \leq \operatorname{var}[\tilde{\eta}]$. Therefore, (3.14) is a variability ordering. 
Theorem 3.3. Consider two germ-grain models with the same typical grains $X_{0}$ such that $N$ and $\tilde{N}$ are Neyman-Scott processes with cluster sizes $\eta$ and $\tilde{\eta}$, respectively, and the same cluster point distribution $V$. If $N$ and $\tilde{N}$ have the same intensity and $\eta<_{1-\mathrm{g}} \tilde{\eta}$, then $Z<_{h-B} \tilde{Z}$.

Proof. By Proposition 3.1, it suffices to show that

$$
\mathrm{P}\left(d_{B}\left(x+t u, Y_{0}\right)>t\right) \geq \mathrm{P}\left(d_{B}\left(x+t u, \tilde{Y}_{0}\right)>t\right)
$$

for all $x, u \in \mathbb{R}^{d}$ and all $t \geq 0$. Letting $B^{\prime}:=t B+x+t u$ and using definition (3.3) of the distribution of $Y_{0}$, we obtain

$$
\begin{aligned}
\mathrm{E}[\eta] \mathrm{P}\left(Y_{0} \cap B^{\prime}=\varnothing\right) & =\mathrm{E}\left[\sum_{y \in L_{0}} \mathbf{1}\left\{A \cap B^{\prime}=\varnothing\right\} \Gamma\left(\left(L_{0}-y\right) \backslash\{0\}, d A\right)\right] \\
& =\mathrm{P}(\eta=1)+\sum_{n=2}^{\infty} \mathrm{P}(\eta=n) n \int f(y)^{n-1} V(\mathrm{~d} y),
\end{aligned}
$$

where $f(y):=\int \mathrm{P}\left(\left(X_{0}+z-y\right) \cap B^{\prime}=\varnothing\right) V(\mathrm{~d} z)$ and where the second identity comes from a straightforward calculation using the definition of the typical cluster $L_{0}$ of a Neyman-Scott process. By Fubini's theorem, this means that

$$
\mathrm{P}\left(Y_{0} \cap B^{\prime}=\varnothing\right)=\mathrm{E}[\eta]^{-1} \int \mathrm{E}\left[\eta f(y)^{\eta-1}\right] V(\mathrm{~d} y) .
$$

We can now use our assumption (3.14) to derive

$$
\mathrm{P}\left(Y_{0} \cap B^{\prime}=\varnothing\right) \geq \mathrm{E}[\tilde{\eta}]^{-1} \int \mathrm{E}\left[\tilde{\eta} f(y)^{\tilde{\eta}-1}\right] V(\mathrm{~d} y) .
$$

Reversing the above steps, we get (3.17) and, hence, the asserted result.

Our next result shows that the assumptions of Theorem 3.3 do not only entail the empty space hazard ordering but also a larger variability of $\tilde{N}$.

Proposition 3.4. Consider Neyman-Scott processes $N$ and $\tilde{N}$ as in Theorem 3.3, and assume in addition that $\eta$ and $\tilde{\eta}$ are square integrable. Then

$$
\operatorname{var}[N(B)] \leq \operatorname{var}[\tilde{N}(B)], \quad B \in \mathcal{B}^{d} \text { bounded } .
$$

Proof. Let $B \in \mathscr{B}^{d}$ be bounded. By (3.1),

$$
\begin{aligned}
\operatorname{var}[N(B)] & =\lambda_{\Pi} \int \mathrm{E}\left[\sum_{i=1}^{\eta} \mathbf{1}\left\{Y_{i}+x \in B\right\}\right]^{2} \mathrm{~d} x \\
& =\lambda_{\Pi} \mathrm{E}[\eta] \int \operatorname{var}\left[1\left\{Y_{1}+x \in B\right\}\right] \mathrm{d} x+\lambda_{\Pi} \mathrm{E}\left[\eta^{2}\right] \int\left(\mathrm{E}\left[\mathbf{1}\left\{Y_{1}+x \in B\right\}\right]\right)^{2} \mathrm{~d} x
\end{aligned}
$$

where we can use the second Wald identity (or a direct calculation) to get the second equality. Since $\lambda_{\Pi} \mathrm{E}[\eta]=\tilde{\lambda}_{\tilde{\Pi}} \mathrm{E}[\tilde{\eta}]=\lambda$, inequality (3.18) boils down to (3.16).

Example 3.5. Let $N$ and $\tilde{N}$ be as in Theorem 3.3, and assume that $\eta \equiv 1$. Then $N$ is again a Poisson process with intensity 1 since the points of the original Poisson process $\Pi$ are independently shifted. Let $a \in[0,1]$. Since $\eta_{l} \equiv 0$ and $\mathrm{E}\left[s^{\eta_{l}}\right] \equiv 1$, we have, for each $\tilde{\eta}$, $1 \equiv \eta<_{1-\mathrm{g}} \tilde{\eta}$ and $Z<_{h-B} \tilde{Z}$. This example shows that within the class of Neyman-Scott germ-grain processes with fixed intensity and typical grain the stochastically smallest empty space appears for the Boolean model. 
Example 3.6. Let $N$ and $\tilde{N}$ be as in Theorem 3.3, and assume that $\eta$ and $\tilde{\eta}$ are Poisson distributed with parameters $c$ and $\tilde{c}$, respectively. (Then the clusters are finite Poisson processes.) We have, for any $a \in[0,1]$,

$$
\mathrm{E}[\eta]^{-1} \mathrm{E}\left[\mathbf{1}\{\eta \geq 1\} \eta a^{\eta-1}\right]=\mathrm{e}^{-c(1-a)} .
$$

Therefore, (3.14) (and, hence, $Z<_{h-B} \tilde{Z}$ ) holds if and only if $c \leq \tilde{c}$. Note that, for Poisson distributed $\eta$, the length biased $\eta_{l}$ has the same (Poisson) distribution. Increasing the mean number of Poisson cluster points in a Neyman-Scott germ-grain model while keeping the overall intensity constant does indeed lead to more clustering and stochastically larger empty space.

Example 3.7. Assume that $\eta$ and $\tilde{\eta}$ are binomially distributed with parameters $(n, p)$ and $(\tilde{n}, \tilde{p})$, respectively. Since $g_{\eta}(s)=((1-p)+p s)^{n}$, it follows that (3.14) is equivalent to

$$
((1-p)+p s)^{n-1} \geq((1-\tilde{p})+\tilde{p} s)^{\tilde{n}-1}, \quad s \in[0,1] .
$$

If, for instance, $n=\tilde{n}$ then this inequality is implied by $p \leq \tilde{p}$.

Example 3.8. Assume that $\eta$ and $\tilde{\eta}$ are negative binomially distributed with parameters $(p, r)$ and $(\tilde{p}, \tilde{r})$, respectively. The corresponding length-biased variables $\eta_{l}$ and $\tilde{\eta}_{l}$ are again negative binomially distributed with parameters $(p, r+1)$ and $(\tilde{p}, \tilde{r}+1)$, respectively. For $p=\tilde{p}$, if $r \leq \tilde{r}$ then $\eta<_{1-\mathrm{g}} \tilde{\eta}$, and, for $r=\tilde{r}$, if $p \geq \tilde{p}$ then $\eta<_{1-\mathrm{g}} \tilde{\eta}$. This is a special case of a more general setting. If $\eta=\sum_{i=1}^{\kappa} \vartheta_{i}$ and $\tilde{\eta}=\sum_{i=1}^{\tilde{\kappa}} \tilde{\vartheta}_{i}$ for i.i.d. variables $\left\{\vartheta_{i}\right\}_{i \geq 1}$, independent of $\kappa$ (all variables taking on natural values), then $\vartheta_{i}<_{1-\mathrm{g}} \tilde{\vartheta}_{i}$, and $\kappa<_{1-\mathrm{g}} \tilde{\kappa}$ implies that $\eta<_{1-\mathrm{g}} \tilde{\eta}$.

Our next result generalises Theorem 5.4 of [12].

Theorem 3.4. Consider two germ-grain models with the same typical grains $X_{0}$ based on Gauss-Poisson processes $N$ and $\tilde{N}$. Assume that $N$ and $\tilde{N}$ have the same intensity and that the probabilities $p$ and $\tilde{p}$ for having secondary points, respectively, satisfy $p \leq \tilde{p}$. Then $Z<_{h-B} \tilde{Z}$.

Proof. Fix $t \geq 0$ and $C \in \mathcal{B}^{d}$. From the defining properties of a Gauss-Poisson process (see Example 3.2) we have

$$
\begin{aligned}
\bar{V}_{i, B} K_{i, B}(t, C)= & \frac{1-p}{1+p} \bar{C}_{i, B}\left(\mathbb{R}^{d} \times C\right)+\frac{p}{1+p} \int_{\mathbb{R}^{d} \times C} a(x, u) \bar{C}_{i, B}(\mathrm{~d}(x, u)) \\
& +\frac{p}{1+p} \int_{\mathbb{R}^{d} \times C} b(x, u) \bar{C}_{i, B}(\mathrm{~d}(x, u)),
\end{aligned}
$$

where

$$
a(x, u):=\mathrm{P}\left(d_{B}\left(x+t u, Y+X_{0}\right)>t\right), \quad b(x, u):=\mathrm{P}\left(d_{B}\left(x+t u,-Y+X_{0}\right)>t\right),
$$

and $Y$ and $X_{0}$ are independent. Therefore, by Proposition 3.1, it suffices to show that

$$
\frac{1-p}{1+p}+\frac{p a}{1+p}+\frac{p b}{1+p} \geq \frac{1-\tilde{p}}{1+\tilde{p}}+\frac{\tilde{p} a}{1+\tilde{p}}+\frac{\tilde{p} b}{1+\tilde{p}}
$$

for all $a, b \in[0,1]$. Simple algebra shows that this inequality is equivalent to

$$
2 \tilde{p}-a \tilde{p}-b \tilde{p} \geq 2 p-a p-b p .
$$

The latter is implied by our assumption $p \leq \tilde{p}$. 
Proposition 3.5. Consider Gauss-Poisson processes $N$ and $\tilde{N}$ as in Theorem 3.4. Then (3.18) holds.

Proof. By (3.1), we obtain, for bounded $B \in \mathcal{B}^{d}$,

$$
\begin{aligned}
\operatorname{var}[N(B)] & =\lambda_{\Pi}(1-p) \int \mathbf{1}\{x \in B\} \mathrm{d} x+\lambda_{\Pi} p \int \mathrm{E}\left[(\mathbf{1}\{x \in B\}+\mathbf{1}\{Y+x \in B\})^{2}\right] \mathrm{d} x \\
& =\lambda_{\Pi}(1+p) V_{d}(B)+\lambda_{\Pi} p \int_{B} \mathrm{P}(Y+x \in B) \mathrm{d} x .
\end{aligned}
$$

As $\lambda_{\Pi}(1+p)=\lambda_{\tilde{\Pi}}(1+\tilde{p})=\lambda$, the result follows from the monotonicity of $p /(1+p)$.

In our next result we will multiply each point of the typical cluster $L_{0}$ of $N$ with a random variable $W \in(0,1]$, i.e. we assume that the typical cluster $\tilde{L}_{0}$ of $\tilde{N}$ has the distribution of $W L_{0}=\left\{W x: x \in L_{0}\right\}$. Compared with $\tilde{L}_{0}$, the points of $L_{0}$ are more spread out. Note that we allow any sort of dependence between $L_{0}$ and $W$.

Proposition 3.6. Consider two Poisson cluster processes $N$ and $\tilde{N}$ based on the same Poisson process $\Pi$ and typical clusters $L_{0}$ and $\tilde{L}_{0}$, respectively. Assume that $\tilde{L}_{0}$ is distributed as $W L_{0}$ for some random variable $W \in(0,1]$. Then $Z<_{h-B} \tilde{Z}$.

Proof. Take $x_{1}, \ldots, x_{n} \in \mathbb{R}^{d}$, and set $\psi:=\left\{x_{j}: j=1, \ldots, n\right\}$. Let $w \leq 1$, and define $\tilde{\psi}:=\left\{w x_{j}: j=1, \ldots, n\right\}$. Let $u \in \partial B^{*}$ and $t \geq 0$. In view of (3.6) it is sufficient to show that

$$
\mathbf{1}\left\{\left(\left(\tilde{\psi}-w x_{i}\right) \backslash\{0\}\right) \cap(t u+t B)=\varnothing\right\} \leq \mathbf{1}\left\{\left(\left(\psi-x_{i}\right) \backslash\{0\}\right) \cap(t u+t B)=\varnothing\right\}
$$

holds for any $i \in\{1, \ldots, n\}$. Assume that the left-hand side of (3.19) equals 1. This is equivalent to $w\left(x_{j}-x_{i}\right) \notin t u+t B$ for all $j \neq i$. Since $u \in \partial B^{*}$, we have $0 \in \partial(t u+t B)$. Therefore, the convexity of $t u+t B$ and $w \leq 1$ imply that also $x_{j}-x_{i} \notin t u+t B$ for all $j \neq i$. This shows (3.19) and, hence, the proposition.

Remark 3.4. The assumptions of Proposition 3.6 do not imply the variance ordering (3.18). However, we may consider the variance of the random variable $\int f \mathrm{~d} N$, where $f(x):=$ $\mathbf{1}\{\|x\| \leq r\}\|x\|^{-\alpha}, r>0$ and $1 \leq 2 \alpha<d$. By the functional version of (3.1),

$$
\begin{aligned}
\operatorname{var}\left[\int f \mathrm{~d} \tilde{N}\right] & =\lambda_{\Pi} \mathrm{E}\left[\int\left(\int f(x+W y) L_{0}(\mathrm{~d} y)\right)^{2} \mathrm{~d} x\right] \\
& =\lambda_{\Pi} \mathrm{E}\left[W \int\left(\int f(W z+W y) L_{0}(\mathrm{~d} y)\right)^{2} \mathrm{~d} z\right] \\
& =\lambda_{\Pi} \mathrm{E}\left[W^{1-2 \alpha} \int\left(\int \mathbf{1}\{W\|z+y\| \leq r\}\|z+y\|^{-\alpha} L_{0}(\mathrm{~d} y)\right)^{2} \mathrm{~d} z\right] \\
& \geq \lambda_{\Pi} \mathrm{E}\left[\int\left(\int \mathbf{1}\{\|z+y\| \leq r\}\|z+y\|^{-\alpha} L_{0}(\mathrm{~d} y)\right)^{2} \mathrm{~d} z\right],
\end{aligned}
$$

where we have used the facts that $W \leq 1$ and $W^{1-2 \alpha} \geq 1$. Therefore, $\int f \mathrm{~d} \tilde{N}$ has a greater variance than $\int f \mathrm{~d} N$. The assumption $2 \alpha<d$ and a suitable moment condition on $L_{0}\left(\mathbb{R}^{d}\right)$ guarantee the finiteness of the above integrals. This can be checked with Jensen's inequality and polar coordinates. Also, norms other than the Euclidean norm can be used. 
Recall that a random variable $\tilde{W}$ is stochastically smaller than another random variable $W$ if $\mathrm{P}(\tilde{W}>t) \leq \mathrm{P}(W>t)$ for all $t \geq 0$.

Proposition 3.7. Let $N$ and $\tilde{N}$ be as in Proposition 3.6. Let $L$ be a point process, and assume that $L_{0}$ is distributed as $W L$ for a random variable $W>0$ independent of $L$. Assume that $\tilde{L}_{0}$ is distributed as $\tilde{W} L$ for a random variable $\tilde{W}>0$ independent of $L$. If $\tilde{W}$ is stochastically smaller than $W$ then $Z<_{h-B} \tilde{Z}$.

Proof. By inverse coupling based on a uniformly distributed random variable that is independent of $L$ we can assume that $(W, \tilde{W})$ is independent of $L$ and that $\tilde{W} \leq W$ everywhere on the underlying probability space. Since $\tilde{L}_{0}=\tilde{W} / W L_{0}$, we can apply Proposition 3.6 with $W$ replaced by $\tilde{W} / W \leq 1$.

\section{Mixed Poisson germ-grain models}

In this section we consider a germ-grain model $Z$ based on a mixed Poisson process $N$. This means that there is a random variable $\Lambda \geq 0$ such that the conditional distribution of $N$ given $\Lambda$ is that of a stationary Poisson process with intensity $\Lambda$. We assume that $E[\Lambda]$ (the intensity of $N$ ) is positive and finite.

It is convenient to use the notation

$$
R_{B}(t)=\mathrm{E}\left[V_{d}\left(X_{0}+t B^{*}\right)\right], \quad t \geq 0 .
$$

Theorem 4.1. The B-relative empty space hazard of a mixed Poisson germ-grain model with compact, convex grains is given by

$$
r_{B}(t, C)=\sum_{i=0}^{d-1}(d-i) t^{d-i-1} b_{d-i} \mathrm{E}\left[\exp \left[-\Lambda R_{B}(t)\right]\right]^{-1} \mathrm{E}\left[\Lambda \exp \left[-\Lambda R_{B}(t)\right]\right] \bar{S}_{i, B}(t, C) .
$$

Proof. Again, we will use (2.14). To do so, we note that the Palm probability measure $\mathrm{P}_{N}^{0}$ of a mixed Poisson process $N$ satisfies

$$
\mathrm{P}_{N}^{0}((\Lambda, N) \in \cdot)=\mathrm{E}[\Lambda]^{-1} \mathrm{E}[\Lambda \mathbf{1}\{(\Lambda, N \cup\{0\}) \in \cdot\}] .
$$

This formula can be derived by conditioning and using the properties of a Poisson process. Since, moreover, the conditional distribution $\mathrm{P}_{N}^{0}\left(Z^{!} \in \cdot \mid \Lambda\right)$ (cf. (2.13) for the definition of the random set $Z^{!}$) is that of a Boolean model with germ intensity $\Lambda$, we obtain, for all $(x, u) \in \mathbb{R}^{d} \times \mathbb{R}^{d}$,

$$
\begin{aligned}
\mathrm{P}_{N}^{0}\left(d_{B}\left(x+t u, Z^{!}\right)>t\right) & =\mathrm{E}[\Lambda]^{-1} \mathrm{E}\left[\Lambda \mathrm{P}\left(d_{B}(x+t u, Z)>t \mid \Lambda\right)\right] \\
& =\mathrm{E}[\Lambda]^{-1} \mathrm{E}\left[\Lambda \mathrm{P}\left(d_{B}(0, Z)>t \mid \Lambda\right)\right] \\
& =\mathrm{E}[\Lambda]^{-1} \mathrm{E}\left[\Lambda \exp \left[-\Lambda R_{B}(t)\right]\right],
\end{aligned}
$$

where we have used (2.7) to obtain the last identity. Again, by conditioning and (2.7), we have $1-F_{B}(t)=\mathrm{E}\left[\exp \left[-\Lambda R_{B}(t)\right]\right]$. Inserting our findings into the general formula (2.14) yields assertion (4.1).

In order to state some stochastic ordering consequences of Theorem 4.1, we introduce a stochastic order using cumulants. We say that two nonnegative random variables $\Lambda$ and $\tilde{\Lambda}$ are ordered in the first cumulant order and write $\Lambda<_{\text {cum }} \tilde{\Lambda}$ if, for the corresponding cumulant 
generating functions $C_{\Lambda}$ and $C_{\tilde{\Lambda}}$ taking the first derivatives, we have $C_{\Lambda}^{\prime}(s) \geq C_{\tilde{\Lambda}}^{\prime}(s), s \leq 0$. Note that $\Lambda<_{\text {cum }} \tilde{\Lambda}$ is equivalent to

$$
\mathrm{E}[\exp [-\Lambda s]]^{-1} \mathrm{E}[\Lambda \exp [-\Lambda s]] \geq \mathrm{E}[\exp [-\tilde{\Lambda} s]]^{-1} \mathrm{E}[\tilde{\Lambda} \exp [-\tilde{\Lambda} s]], \quad s \geq 0 .
$$

The left-hand side of (4.2) is the negative logarithmic derivative of the Laplace transform $s \mapsto \mathrm{E}[\exp [-\Lambda s]]$. It is also the hazard rate of the distribution function $G_{\Lambda}$, defined by

$$
G_{\Lambda}(s):=1-\mathrm{E}[\exp [-\Lambda s]], \quad s \geq 0 .
$$

This is a mixture of exponential distributions. Equation (4.2) then means that the corresponding variables are ordered in the hazard rate order, i.e. $G_{\Lambda}<_{h} G_{\tilde{\Lambda}}$. Note that, for $\Lambda$ and $\tilde{\Lambda}$ with equal expected values, $\Lambda<$ cum $\tilde{\Lambda}$ implies that $\operatorname{var}[\Lambda] \leq \operatorname{var}[\tilde{\Lambda}]$; therefore, similarly to the relation ' $<_{1-\mathrm{g}}$ ', the relation ' $<_{\text {cum }}$ ' is a variability ordering in the case of a fixed mean.

An immediate consequence of Theorem 4.1 is the following counterpart of Theorems 3.3 and 3.4. We use similar notation. Again, intuitively speaking, more variability in the mixed Poisson model results in a stochastically greater empty space (a stochastically larger clustering).

Theorem 4.2. Consider two germ-grain models with the same typical grains $X_{0}$ based on mixed Poisson processes $N$ and $\tilde{N}$ with random intensities $\Lambda$ and $\tilde{\Lambda}$, respectively. Assume that $\Lambda<_{\text {cum }} \tilde{\Lambda}$. Then $Z<_{h-B} \tilde{Z}$.

Remark 4.1. If $\Lambda<_{\text {cum }} \tilde{\Lambda}$ and $\mathrm{E}[\Lambda]=\mathrm{E}[\tilde{\Lambda}]$, then (3.18) holds. This is in pleasing accordance with Propositions 3.5 and 3.4 and with Remark 3.4. Inequality (3.18) is a well-known consequence of $\operatorname{var}[\Lambda] \leq \operatorname{var}[\tilde{\Lambda}]$ and the identity

$$
\operatorname{var}[N(B)]=\operatorname{var}[\mathrm{E}[N(B) \mid \Lambda]]+\mathrm{E}[\operatorname{var}[N(B) \mid \Lambda]]=\operatorname{var}[\Lambda(B)]+\mathrm{E}[\Lambda(B)] .
$$

Example 4.1. Let $N$ and $\tilde{N}$ be as in Theorem 4.1, and assume that $\Lambda$ is gamma distributed with shape and scale parameters $\alpha>0$ and $\beta>0$, respectively. This means that $\Lambda$ has density $\beta^{\alpha} \Gamma(\alpha)^{-1} x^{\alpha-1} \exp [-\beta x]$. The Laplace transform of $\Lambda$ can be computed as

$$
\mathrm{E}[\exp [-s \Lambda]]=\frac{\beta^{\alpha}}{(\beta+s)^{\alpha}},
$$

while an equally easy calculation gives

$$
\mathrm{E}[\Lambda \exp [-s \Lambda]]=\frac{\alpha}{\beta+s} \frac{\beta^{\alpha}}{(\beta+s)^{\alpha}} .
$$

Assume now that $\tilde{\Lambda}$ is gamma distributed with parameters $\tilde{\alpha}$ and $\tilde{\beta}$, respectively. Then assumption (4.2) means that $\alpha /(\beta+s) \geq \tilde{\alpha} /(\tilde{\beta}+s)$ holds for all $s \geq 0$. This is equivalent to

$$
\alpha \geq \tilde{\alpha}, \quad \frac{\alpha}{\beta} \geq \frac{\tilde{\alpha}}{\tilde{\beta}} .
$$

Depending on whether or not $\beta \geq \tilde{\beta}$, only one of these equations is relevant. By Theorem 4.2, (4.3) implies the empty space hazard ordering (2.5). Assume, for instance, that $\tilde{\Lambda}$ is exponentially distributed with mean 1 , i.e. $\tilde{\alpha}=\tilde{\beta}=1$, and assume furthermore that $\Lambda$ has also mean 1 , that is, $\alpha=\beta$. Then (4.3) is equivalent to $\alpha \geq \tilde{\alpha}$. Note that the variance of $\Lambda$ satisfies $\operatorname{var}[\Lambda]=\alpha / \beta^{2}=1 / \beta \leq \operatorname{var}[\tilde{\Lambda}]=1$ if $\alpha \geq 1$.

As in the Poisson cluster case, it follows that the relative empty space hazard of a Boolean model is greater than that of a mixed Poisson germ-grain model with the same germ intensity. 
Corollary 4.1. Assume that $Z$ is a Boolean model with typical convex, compact grains distributed as $X_{0}$, and that $\tilde{Z}$ is a mixed Poisson germ-grain model with equal intensity, and also with typical grains distributed as $X_{0}$. Then $Z<_{h-B} \tilde{Z}$.

Proof. Let $\lambda$ denote the germ intensity of the Boolean model $Z$, and let $\tilde{\Lambda}$ be the random intensity of the mixed Poisson process underlying $\tilde{Z}$. It is assumed that $\mathrm{E}[\tilde{\Lambda}]=\lambda$. We check that condition (4.2) holds with $\Lambda \equiv \lambda$. This condition means that

$$
\lambda \mathrm{E}[\exp [-\tilde{\Lambda} s]] \geq \mathrm{E}[\tilde{\Lambda} \exp [-\tilde{\Lambda} s]], \quad s \geq 0 .
$$

In other words, the covariance between $\tilde{\Lambda}$ and $-\exp [-\tilde{\Lambda} s]$ has to be nonnegative. This fact follows from the well-known fact that a single random variable is associated; see [6].

For completeness, we provide the mixed Poisson analogue of Corollary 3.3. The result can be found in Section 3.8 of [7] for the more general case of stationary Cox processes with an absolutely continuous intensity measure. Our proof can be extended to arbitrary stationary Cox processes.

Proposition 4.1. Under the assumptions of Corollary 4.1,

$$
\mathrm{P}(0 \in Z) \geq \mathrm{P}(0 \in \tilde{Z}) .
$$

Proof. Conditioning and (2.8) yield

$$
1-\mathrm{P}(0 \in \tilde{Z})=\mathrm{E}\left[\exp \left[-\Lambda \mathrm{E}\left[V_{d}\left(X_{0}\right)\right]\right]\right] .
$$

By Jensen's inequality, this is bounded from below by $\exp \left[-\lambda \mathrm{E}\left[V_{d}\left(X_{0}\right)\right]\right]$. This lower bound is just $1-\mathrm{P}(0 \in Z)$.

\section{Concluding remarks}

We have derived empty space orderings of Poisson cluster and mixed Poisson germ-grain models. It would be worthwhile to also study other classes of germ processes. Moreover, inequality (3.18) suggests that there might be a more general variability ordering for germ processes, implying the ordering of all relative empty space hazards. A related task is to find a good notion of spread out for a finite point process (with respect to the origin). The point process versions of Proposition 3.4 and Proposition 3.6 should be both special cases of the same principle. Moreover, we believe that Proposition 3.6 has a germ-grain counterpart.

In this paper we have always fixed the distribution of the typical grain. However, it would be quite interesting to study the variability of empty space in germ-grain models for a fixed germ process but variable grain distribution. For instance, one might compare models with equal expected volumes of the typical grains. To illustrate this task, we give one example that is closely related to some of the results in [21].

Example 5.1. Let $X_{0}$ be a random convex body such that $\mathrm{E}\left[V_{d}\left(X_{0}+K\right)\right]$ is finite for all compact $K \subset \mathbb{R}^{d}$. Let $R$ and $\tilde{R}$ be positive random variables with a finite $d$ th moment, and assume that $X_{0}$ and $R$ (respectively $X_{0}$ and $\tilde{R}$ ) are independent. Consider two Boolean models $Z$ and $\tilde{Z}$ based on the same Poisson process $N$ and typical grains $R X_{0}$ and $\tilde{R} X_{0}$, respectively. If

$$
\mathrm{E}\left[R^{i}\right] \geq \mathrm{E}\left[\tilde{R}^{i}\right], \quad i=1, \ldots, d-1,
$$

then (2.5) holds for all structuring elements $B$ such that $X_{0}$ and $B^{*}$ are a.s. in general relative 
position, and all Borel sets $C \subset \mathbb{R}^{d}$. This follows from (2.15) and the scaling property

$$
C_{i}\left(a X_{0} ; B ; \mathbb{R}^{d} \times C\right)=a^{i} C_{i}\left(X_{0} ; B ; \mathbb{R}^{d} \times C\right), \quad a>0 ;
$$

see, e.g. [17] for the Euclidean case $B=B(1)$.

\section{Acknowledgements}

The first author is very grateful for the hospitality of the Mathematical Institute of the University of Wrocław. Large parts of this paper were written while he was visiting the institute. Both authors wish to thank two anonymous referees and the Editor, whose constructive criticisms helped to improve the presentation of the results.

\section{References}

[1] Baddeley, A. ANd Gill, R. D. (1994). The empty space hazard of spatial pattern. Preprint 845, Department of Mathematics, University o Utrecht.

[2] Baddeley, A. ANd Gill, R. D. (1997). Kaplan-Meier estimators of distance distributions for spatial point processes. Ann. Statist. 25, 263-292.

[3] Bordenave, C. And Torrisi, G. L. (2007). Large deviations of Poisson cluster processes. Stoch. Models 23, 593-625.

[4] Chiu, S. N. and Stoyan, D. (1998). Estimators of distance distributions for spatial patterns. Statist. Neerlandica 52, 239-246.

[5] Daley, D. J. And Vere-Jones, D. (2003). An Introduction to the Theory of Point Processes, Vol. I, 2nd edn. Springer, New York.

[6] Esary, J. D., Proschan, F. and Walkaup, D. W. (1967). Association of random variables, with applications. Ann. Math. Statist. 38, 1466-1474.

[7] Hall, P. (1988). Introduction to the Theory of Coverage Processes. John Wiley, New York.

[8] Hansen, M. B., Baddeley, A. J. ANd Gill, R. D. (1999). First contact distributions for spatial patterns: regularity and estimation. Adv. Appl. Prob. 31, 15-33.

[9] Hug, D. and Last, G. (2000). On support measures in Minkowski spaces and contact distributions in stochastic geometry. Ann. Prob. 28, 796-850.

[10] Hug, D., Last, G. AND WeIL, W. (2002). Generalized contact distributions of inhomogeneous Boolean models. Adv. Appl. Prob. 34, 21-47.

[11] Hug, D., LASt, G. AND WeIL, W. (2002). A survey on contact distributions. In Morphology of Condensed Matter (Lecture Notes Phys. 600), eds K. Mecke and D. Stoyan, Springer, Berlin, pp. 317-357.

[12] Last, G. and Holtmann, M. (1999). On the empty space function of some germ-grain models. Pattern Recognition 32, 1587-1600.

[13] Last, G. AND Schassberger, R. (1998). On the distribution of the spherical contact vector of stationary grain models. Adv. Appl. Prob. 30, 36-52.

[14] Molchanov, I. (1997). Statistics of the Boolean model for Practitioners and Mathematicians. John Wiley, Chichester.

[15] Molchanov, I. (2005). Theory of Random Sets. Springer, London.

[16] Müller, A. And Stoyan, D. (2002). Comparison Methods for Stochastic Models and Risks. John Wiley, Chichester.

[17] SchneIder, R. (1993). Convex Bodies: The Brunn-Minkowski Theory (Encyclopedia Math. Appl. 44). Cambridge University Press.

[18] SchneIder, R. AND WeIL, W. (2008). Stochastic and Integral Geometry. Springer, Berlin.

[19] Stoyan, D. (1983). Comparison Methods for Queues and Other Stochastic Models. John Wiley, Chichester.

[20] Stoyan, D., Kendall, W. S. And Mecke, J. (1995). Stochastic Geometry and Its Applications, 2nd edn. John Wiley, Chichester.

[21] Stoyan, H. and Stoyan, D. (1980). On some partial orderings of random closed sets. Math. Operationsforsch. Statist. Ser. Optimization 11, 145-154.

[22] Szekli, R. (1995). Stochastic Ordering and Dependence in Applied Probability (Lecture Notes Statist. 97). Springer, New York.

[23] Van Lieshout, M. N. M. And Baddeley, A. J. (1996). A nonparametric measure of spatial interaction in point patterns. Statist. Neerlandica 50, 344-361. 